\title{
LIDOCAÍNA INTRAVENOSA COMO ALTERNATIVA ANALGÉSICA EM OVINO: ESTUDO DE CASO
}

Vivian Fernanda Barbosa ${ }^{1}$, Vinícius de Jesus Moraes ${ }^{2}$, Karina Médici Madureira ${ }^{1}$, Rodrigo Freitas Bittencourt ${ }^{1}$, Maristela de Cássia Seudo Lopes ${ }^{1}$

1Professor doutor, Universidade Federal da Bahia, (vivian.fernanda@ufba.br) Salvador, BA, Brasil.

2Residente em Anestesiologia e Medicina de Emergência Veterinária, Área Profissional da Saúde, UFBA. Bolsista MEC.

Recebido em: 08/09/2015 - Aprovado em: 14/11/2015 - Publicado em: 01/12/2015 DOI: http://dx.doi.org/10.18677/Enciclopedia_Biosfera_2015_187

Foi atendido em um Hospital Veterinário Universitário, um ovino, raça Santa Inês, três anos de idade, macho, não castrado, $110 \mathrm{~kg}$, com histórico de urolitíase obstrutiva recorrente, claudicação e uso contínuo de anti-inflamatório não esteroide (AINE). Ao exame físico notou-se mucosas avermelhadas, ausência de movimentos ruminais, retração do vazio do flanco e borborigmos em abomaso e intestino. A palpação intestinal revelou fezes com muco abundante, pastosas e verde-escuras. Constatou-se aumento de volume na articulação úmero-radio-ulnar esquerda com dor à palpação e claudicação ao marchar, FC de 126 batimentos/minuto, $f$ de 64 movimentos/minuto, PAS, PAM e PAD de 132, 102 e $87 \mathrm{mmHg}$, respectivamente, turgor cutâneo de 4 a 5 segundos e TPC de 3 segundos. Os achados laboratoriais e de imagem revelaram urolitíase uretral com uremia e osteoartrite na articulação úmero-radio-ulnar esquerda. Instituiu-se tratamento de suporte com interrupção do AINE e como recurso analgésico alternativo, utilizou-se cloridrato de lidocaína (3 $\mathrm{mg} / \mathrm{kg} / \mathrm{h}$ ), durante 3 horas, uma vez ao dia, durante 3 dias. Ao terceiro dia o animal foi encaminhado à uretrostomia, com melhora do estado geral, alimentando-se em pequenas quantidades, com mucosas róseas, 2 movimentos ruminais completos a cada 3 minutos, $f$ de 28 movimentos/minuto, FC de 86 batimentos/minuto e PAS, PAM e PAD de 125, 96 e $82 \mathrm{mmHg}$, respectivamente. Conclui-se que a lidocaína intravenosa contribuiu para a evolução positiva do animal ao ofertar analgesia adicional e melhora da atividade gastrintestinal, sendo recomendada como alternativa ao uso de AINE na espécie ovina.

PALAVRAS-CHAVE: analgesia intravenosa, anestésico local, ruminantes.

\section{INTRAVENOUS LIDOCAINE AS AN ANALGESIC ALTERNATIVE IN SHEEP: A CASE REPORT}

\begin{abstract}
A sheep, Santa Ines, three years old, male, non-neutered, $110 \mathrm{~kg}$, was examined at a veterinary teaching hospital, with a history of recurrent obstructive urolithiasis, lameness and continuous use of nonsteroidal anti-inflammatory (NSAID). Physical examination showed reddish mucous membranes, absence of ruminal movements, retraction of empty flank and borborygmus in abomasum and intestine. Intestinal
\end{abstract}


palpation revealed abundant stools with mucus, pasty and dark green. Swelling in the left humerus, radio and ulna joint with pain on palpation and lameness while marching were verified, HR of 126 beats / min, $f$ of 64 breaths / minute, SBP, MBP and DBP of 132, 102 and $87 \mathrm{mmHg}$, respectively, skin turgor 4 to 5 seconds and 3 seconds capillary refill time were found. Laboratory and imaging findings revealed urethral urolithiasis with uremia and osteoarthritis in the left humerus, radio and ulna joint. Support treatment was initiated, NSAID was aborted and as alternate analgesic was used lidocaine hydrochloride ( $3 \mathrm{mg} / \mathrm{kg} / \mathrm{h}$ ) for 3 hours, once a day for 3 days. On the third day the animal was submitted to urethrostomy with general improvement, feeding in small amounts, with rosy mucous, 2 full ruminal movements every 3 minutes, $f$ of 28 breaths / minute, HR of 86 beats / minute, SBP, MBP and DBP of 125, 96 and $82 \mathrm{mmHg}$, respectively. Intravenous lidocaine contributed to the positive evolution of the animal to offer additional analgesia and improves gastrointestinal activity. This protocol is recommended as an alternative to NSAID use in sheep.

KEYWORDS: intravenous analgesia, local anesthetic, ruminants.

\section{INTRODUÇÃO}

O controle inadequado da dor denota reconhecido potencial deletério em humanos e animais (LIZARRAGA \& CHAMBERS, 2012). A privação analgésica confronta preceitos éticos de bem-estar e está associada a graves perdas econômicas no âmbito produtivo (GALATOS, 2011; WINTER, 2008).

Em animais, o reconhecimento de condições dolorosas tem sido prioritariamente baseado em fatores objetivos que incluem alterações em parâmetros cardiovasculares como frequência cardíaca, pressão arterial, concentrações séricas de beta-endorfinas, catecolaminas e corticosteroides, associados à interpretação de variações comportamentais. Os ruminantes são aparentemente estoicos e, na qualidade de presa, expressam reações comportamentais sutis, fato que dificulta o reconhecimento e a quantificação da dor, sobretudo em ovinos (FITZPATRICK et al., 2006; VALVERDE \& GUNKEL, 2005). Nessa espécie, dentre as manifestações comportamentais mais comuns face a dor, destacam-se inapetência, ranger de dentes, tremores labiais, pressionar a cabeça, isolamento, imobilidade, movimentos de cavar e marcha anormal (GALATOS, 2011; LIZARRAGA \& CHAMBERS, 2012).

No âmbito das condições potencialmente álgicas, os ovinos estão sujeitos a procedimentos realizados a campo tais como castração, cesariana e corte da cauda, estão susceptíveis a desenvolver mastite, laminite e artropatias, além de serem frequentemente usados com propósitos educacionais e em pesquisas biomédicas, as quais usualmente envolvem procedimentos cirúrgicos invasivos (LIZARRAGA \& CHAMBERS, 2012).

Todavia, a instituição de terapia analgésica na espécie é corriqueiramente negligenciada ou insuficiente, em decorrência de fatores como a dificuldade em reconhecer e avaliar a dor; a falta de conhecimento sobre o uso adequado de analgésicos; o medo de resíduos e por limitações financeiras (VALVERDE \& GUNKEL, 2005).

Dentre as modalidades farmacológicas disponíveis, os anti-inflamatórios não esteroides (AINE) representam o recurso analgésico mais comumente acessado por criadores e produtores rurais, porém, quando administrados em altas doses ou por 
períodos prolongados não raramente associam-se a efeitos colaterais significativos como ulceração abomasal e toxicidade renal (SMITH et al., 2008; GALATOS, 2011).

Pacientes com histórico de alterações gástricas ou renais, associadas a quadros dolorosos, podem apresentar deterioração orgânica expressiva e representam uma importante parcela na qual o uso de AINE apresenta-se contraindicado, porém, na absoluta maioria dos casos, a abolição ou amenização do estado álgico torna-se fundamental à evolução positiva do estado clínico (SMITH et al., 2008).

A lidocaína é o anestésico local mais largamente usado, titulado protótipo dos derivados aminoamidas. É considerado um fármaco versátil, utilizado para fins diversos em medicina humana e veterinária, incluindo o controle da dor visceral, central e neuropática; anestesia local e loco-regional e na terapia antiarrítmica (GOLZARI et al., 2014).

A ação multimodal da lidocaína por via intravenosa (IV) é periférica e central e envolve mecanismos como bloqueio de canais de sódio, ação glicinérgica, bloqueio de receptores n-metil-d-aspartato, agonismo muscarínico, redução de aminoácidos excitatórios, liberação de opioides endógenos e redução da substância $P$. Em baixas concentrações, a lidocaína inibe a atividade anormal em fibras aferentes primárias, principalmente fibras $\mathrm{C}$, causa bloqueio simpático, vasodilatação e quebra do círculo vicioso que mantém a dor (DE OLIVEIRA et al., 2010; LAURENT, 2008).

O uso da lidocaína IV cresceu extensivamente nas últimas décadas, objetivando-se sua efetividade analgésica multimodal em múltiplas espécies sob condições dolorosas diversas (DUKE, 2013; GOLZARI et al., 2014; GOZALOMARCILLA et al., 2014; NANNARONE et al., 2015; ORTEGA \& CRUZ, 2011; VESAL et al., 2011). Por esta via, esse fármaco possui ação antihiperalgésica, ameniza a resposta inflamatória à isquemia tecidual e promove efeito analgésico transoperatório, além de suprimir o consumo pós-cirúrgico de opioides (MCCLEANE, 2007; LAURETTI, 2008). Seu efeito torna-se particularmente relevante em condições inflamatórias (DUKE, 2013).

Em humanos, é comumente utilizada no tratamento da dor neuropática, permite administração a curto prazo capaz de produzir analgesia a longo prazo, de baixo custo, com redução ou interrupção de outros analgésicos, além de possibilitar repetição do tratamento sem ocasionar tolerância, mostrando-se de fácil administração (MCCLEANE, 2007).

Em um estudo, foi demonstrado, experimentalmente, que a lidocaína IV produz trêz fases distintas de alívio álgico, sendo que a primeira surge durante a infusão e diminui 30 a 60 minutos após o seu término; a segunda é uma fase transitória que ocorre seis horas após a administração e a terceira aparece em 24 a 48 horas após a infusão contínua e pelos próximos 21 dias (ARAÚJO et al., 2003).

De acordo com a literatura, o uso da lidocaína em equinos tem se mostrado promissor relatando-se como efeitos: diminuição da dor e melhora da função gastrintestinal pós-cirúrgica, ação antihiperalgésica, propriedades anti-inflamatórias, melhora da reabilitação e redução do consumo de anestésicos gerais (GOZALOMARCILLA et al., 2014).

Em cães, a infusão de lidocaína preveniu resposta simpática à estimulação cirúrgica e reduziu o consumo intra-operatório de opioides, sem causar instabilidades cardiorespiratória clinicamente importante (ORTEGA \& CRUZ, 2011). Outros autores referem efeitos relevantes em condições inflamatórias na espécie, 
bem como em felinos, embora nesses últimos o uso de altas doses ou em infusão contínua permaneça controverso (DUKE, 2013).

Existem poucos estudos no que concerne ao uso da lidocaína IV com finalidades analgésicas em ruminantes. VESAL et al. (2011) referem redução do consumo de isofluorano (16,7\%) durante cirurgia umbilical em bezeros com a administração desse anestésico local em bolus (2 $\mathrm{mg} / \mathrm{kg})$ seguido de infusão contínua (50 $\mathrm{mcg} / \mathrm{kg} / \mathrm{min}$.).

Sem dúvida, um dos grandes desafios do manejo da dor em pequenos ruminantes reside na escassez de artigos científicos no que se refere ao uso seguro de modalidades analgésicas promissoras, de modo que a expectativa dos efeitos terapêuticos muitas vezes é pautada na experiência com animais de companhia, seres humanos ou mesmo em outras espécies não ruminantes, fato que corriqueiramente resulta em pacientes subtratados ou até intoxicados (PLUMMER \& SCHLEINING, 2013).

Em face à demanda por informações relevantes sobre recursos analgésicos em pequenos ruminantes, objetiva-se com este relato resgatar o caso de um ovino com quadro doloroso e contraindição à terapia anti-inflamatória, cujo uso da lidocaína IV mostrou-se uma alternativa analgésica promissora.

\section{RELATO DE CASO}

Foi atendido em março de 2015 em um Hospital Veterinário Universitário, um ovino da raça Santa Inês, de três anos de idade, macho, não castrado, $110 \mathrm{~kg}$, com histórico de urolitíase obstrutiva e claudicação. O animal apresentava-se apático, desidratado, com apetite diminuído e dor no membro anterior esquerdo, a qual vinha sendo tratada com cetoprofeno e dipirona associada à escopolamina havia cinco dias. Em adição, o proprietário relatou uso prolongado de fenilbutazona, mas não soube informar período e frequência de administração.

Ao exame físico o animal apresentava mucosas avermelhadas, linfonodos parotídeos reativos, ausência de movimentos ruminais, retração do vazio do flanco devido ao pouco conteúdo gástrico e borborigmos em abomaso e intestino. A avaliação das fezes revelou muco abundante, consistência pastosa e coloração verde-escuras. Constatou-se aumento de volume na articulação úmero-radio-ulnar esquerda com dor à palpação e claudicação ao marchar, frequências cardíaca (FC) e respiratória $(f)$ de 126 batimentos por minuto (bpm) e 64 movimentos por minuto $(\mathrm{mpm})$, respectivamente, pressões arteriais sistólica (PAS), média (PAM) e diastólica (PAD) de 132, 102 e $87 \mathrm{mmHg}$, respectivamente, turgor cutâneo de quatro a cinco segundos e tempo de preenchimento capilar (TPC) de três segundos. A sonda de Folley vesical implantada havia oito dias eliminava por gotejamento urina de coloração avermelhada.

Foram solicitados como exames primários, hemograma e dosagens de uréia, creatinina, aspartato aminotransferase (AST), gama glutamil transferase (GGT), bilirrubina total, direta e indireta, proteínas séricas totais e frações, urinálise e hemogasometria. Os achados laboratoriais revelaram hipoproteinemia sérica $(5,3$ $\mathrm{g} / \mathrm{dL})$ por hipoalbuminemia $(1,8 \mathrm{~g} / \mathrm{dL})$, elevados níveis de uréia $(48,5 \mathrm{mg} / \mathrm{dL})$, creatinina $(2,3 \mathrm{mg} / \mathrm{dL})$, gama glutamil transferase $(83,1 \mathrm{mg} / \mathrm{dL})$ e fribrinogênio $(600$ $\mathrm{mg} / \mathrm{dL}$ ). A urinálise evidenciou, ao exame químico, proteinúria moderada e sangue intenso, com hemácias incontáveis à sedimentoscopia. Na análise hemogasométrica venosa constatou-se $\mathrm{pH}$ de 7,4 , pressões de dióxido de carbono de $23,9 \mathrm{mmHg}$ e bicarbonato de $14,5 \mathrm{mmol} / \mathrm{L}$, quadro compatível com acidose metabólica 
compensada. A imagem radiográfica revelou osteoartrite da articulação úmero-radioulnar esquerda e a ultrassonografia abdominal e pélvica retratou uretra dilatada, com paredes espessadas preenchida com pontos hiperecogênicos compatíveis com cálculos.

Findada a avaliação clínica, obteve-se o diagnóstico de urolitíase obstrutiva e osteoartrite da articulação úmero-radio-ulnar esquerda. Embora não realizado exame endoscópico, mas avaliando-se as informações obtidas no histórico sobre o uso contínuo de AINE, foi considerada também a possibilidade de abomasite.

Como tratamento, optou-se pela estabilização clínica do paciente para posterior agendamento cirúrgico. A terapia consistiu na administração de fluidoterapia com solução de Ringer lactato ${ }^{1}$, visando-se correção do desequilíbrio ácido-básico, ou solução de $\mathrm{NaCl} 0,9 \%{ }^{2}$, usada para diluições de fármacos; administração de cloreto de amônio ${ }^{3}$, como acidificante urinário face ao quadro de urolitíase; cloridrato de ranitidina ${ }^{4}$, para proteção da mucosa abomasal; dipirona associada à escopolamina ${ }^{5}$ como analgésico e anti-espasmódico das vias urinárias; sucralfato $^{6}$, para proteção das mucosas abomasal e duodenal e norfloxacina ${ }^{7}$, visando-se antibióticoterapia preventiva da infecção urinária. Em face à persistência da inapetência e das alterações cardiorrespiratórias e comportamentais compatíveis com dor, optou-se por utilizar, como recurso analgésico adicional, cloridrato de lidocaína $2 \%$ sem vasoconstrictor ${ }^{8}(3 \mathrm{mg} / \mathrm{kg} / \mathrm{h})$ diluída em solução de cloreto de sódio $0,9 \%$ em taxa $10 \mathrm{~mL} / \mathrm{kg} / \mathrm{h}$ durante três horas, uma vez ao dia.

Os parâmetros FC, $f$, temperatura retal (TR) e movimentos ruminais (MR) foram avaliados com intervalos de $4 \mathrm{~h}$ por três dias consecutivos (Tabela 1). Após o término da infusão contínua (IC) de lidocaína constatava-se retorno das variáveis aferidas aos limites normais para espécie, com pequenas oscilações ao longo dos dias. Adicionalmente, pode-se verificar aumento dos movimentos ruminais em paralelo ao aumento do interesse do ovino por alimento, cuja ingestão não foi quantificada, porém houve formação de fezes consistentes com a evolução do tratamento.

TABELA 1 Valores médios ( $\mathrm{x}$ ) e desvios padrão (s) dos três dias de tratamento, em intervalos de quatro horas, das variáveis frequência cardíaca (FC), frequência respiratória $(f)$, temperatura retal (TR) e movimentos ruminais (MR) em ovino, após infusão contínua de lidocaína intravenosa.

\begin{tabular}{ccccccc}
\hline \hline & $\mathbf{4 h}$ & $\mathbf{8 h}$ & $\mathbf{1 2 h}$ & $\mathbf{1 6 h}$ & $\mathbf{2 0 h}$ & $\mathbf{2 4 h}$ \\
\hline \hline FC & $84 \pm 6$ & $88 \pm 11$ & $87 \pm 5$ & $92 \pm 9$ & $87 \pm 8$ & $94 \pm 7$ \\
(bat/min) & & & & & & \\
$f(\mathrm{mov} / \mathrm{min})$ & $36 \pm 3$ & $34 \pm 8$ & $29 \pm 5$ & $29 \pm 6$ & $27 \pm 5$ & $34 \pm 4$ \\
TR (C) & $39,9 \pm 0,5$ & $39,8 \pm 0,3$ & $39,6 \pm 0,5$ & $39,5 \pm 0,4$ & $39,7 \pm 0,3$ & $39,6 \pm 0,4$ \\
MR (mov/ 3 & $2 \pm 1$ & $2 \pm 1$ & $3 \pm 1$ & $2 \pm 1$ & $2 \pm 1$ & $2 \pm 1$ \\
min) & & & & & & \\
\hline \hline
\end{tabular}

1 Solução de ringer com lactato, Lab. Baxter, São Paulo - SP.

2 Solução de cloreto de sódio 0,9\%, Lab. Baxter, São Paulo - SP.

${ }^{3}$ Cloreto de amônio, Lab. Farmos, Rio de Janeiro - RJ

${ }^{4}$ Cloridrato de ranitidina, Lab. Farmace, Barbalha-CE

5 Buscofin Composto $\AA$, Lab. Agener União, Embuguaçu - SP

6 Sulcralfilm®, Lab. EMS Sigma Pharma Ltda., Hortolândia - SP

7 Norflomax $\AA^{\circledR}$, Lab. Ouro fino, Cravinhos - SP

8 Xylestesin ${ }^{\circledR}$, Lab. Cristália, Itapira - SP

ENCICLOPÉDIA BIOSFERA, Centro Científico Conhecer - Goiânia, v.11 n.22; p.2145 
A avaliação laboratorial denotou aumento da creatinina dos valores iniciais (2,3 $\mathrm{mg} / \mathrm{dL})$ para $2,5 \mathrm{mg} / \mathrm{dL}$ e diminuição da albumina $(1,8 \mathrm{~g} / \mathrm{dL})$ para $1,6 \mathrm{~g} / \mathrm{dL}$, no entanto, o fibrinogênio reduziu para $400 \mathrm{mg} / \mathrm{dL}$. Houve melhora das variáveis relacionadas à hidratação, como redução do tempo de turgor cutâneo e do TPC.

Ao terceiro dia de tratamento o animal foi encaminhado ao centro cirúrgico para uretrostomia, como aparente melhora do estado geral, alimentando-se em pequenas quantidades constatando-se mucosas róseas, dois movimentos ruminais completos a cada três minutos, $\mathrm{T}^{\mathrm{C}} \mathrm{C}$ de $39,2^{\circ} \mathrm{C}, f$ de $28 \mathrm{mpm}, \mathrm{FC}$ de 86 bmp e PAS, PAM e PAD de 125, 96 e $82 \mathrm{mmHg}$, respectivamente.

\section{DISCUSSÃO}

Afecções dolorosas são relativamente comuns em animais de produção, embora 0 reconhecimento e a quantificação da dor sejam comumente negligenciados (VALVERDE \& GUNKEL, 2005). Nesse interim, a instituição de terapia analgésica adequada e eficaz tem representado importante desafio, sobretudo pela falta de recursos analgésicos acessíveis e alternativos.

O uso indiscriminado de AINE é comum em animais de produção, seja pelo fácil acesso à modalidade medicamentosa, pelo baixo custo de algumas apresentações farmacêuticas ou pela eficácia analgésica e anti-inflamatória da maioria desses agentes. Contudo, alguns representantes dessa classe são menos seletivos e efetuam o bloqueio da enzima cicloxigenase-1, interferindo com habilidades fisiológicas de manutenção da função renal e da integridade das mucosas gástricas e intestinais. Tais efeitos são reportados durante a administração a médio e longo prazo em pacientes hígidos, mas podem agravar rapidamente quadros prévios de insuficiência renal ou apresentar-se deletério a curto prazo em pacientes sensíveis (SMITH et al., 2008; GALATOS, 2011).

O ovino foco desse relato foi tratado previamente e permanecia recebendo AINE como recurso analgésico, fato que poderia estar associado ou representou fator agravante ao quadro de insuficiência renal e às alterações gastrintestinais e metabólicas constatadas em sua admissão hospitalar. Adicionalmente, o animal apresentava-se inapetente, apático, isolado e com sinais de dor articular resistente à medicação.

Após a instituição da terapia clínica, visando-se supressão do quadro gástrico e melhora da função renal, não houve evolução significativa do paciente, no qual persistiam as alterações cardiorrespiratórias e comportamentais associadas à dor.

Dentre do contexto clínico de quadro ostearticular doloroso associado à condição urêmica, optou-se pela utilização da lidocaína como recurso analgésico alternativo ao uso de AINE, objetivando-se proporcionar analgesia e, desta forma, contribuir para a melhora do estado geral do paciente.

Iniciou-se a IC de lidocaína sem vasoconstritor na taxa de $3 \mathrm{mg} / \mathrm{kg} / \mathrm{h}$ por $3 \mathrm{~h}$ com repetições a cada $24 \mathrm{~h}$. A escolha da dose de infusão foi baseada no estudo de VESAL et al (2011) em bezerros, contudo optou-se por não se utilizar bolus prévio à infusão em virtude do prejuízo renal instalado, o qual poderia comprometer a excreção do fármaco por tal via, predispondo o animal à intoxicação. Considerandose tal premissa, decidiu-se por uma única infusão diária de três horas, já que MCCLEANE (2007) refere longa eficácia analgésica com a administração IV desse anestésico local a curto prazo associada a ausência de tolerância. ARAUJO et al. (2003) reforçam a assertiva ao alegarem que a infusão intravenosa suprime a dor durante a administração, seis, 24 e 48 horas após esta e pelos próximos 21 dias. 
Em equinos com cólica submetidos à laparotomia, a aplicação de dose bolus de lidocaína $(1,5 \mathrm{mg} / \mathrm{kg})$ prévio à infusão intravenosa $(50 \mathrm{mcg} / \mathrm{kg} / \mathrm{min}$.) não denotou efeitos cardiorrespiratórios nem influenciou no requerimento de isofluorano ou na qualidade da recuperação, a qual foi considerada boa ou execelente em $86 \%$ dos equinos avaliados. Desta forma, os autores consideraram desnecessário o uso do bolus préviamente à infusão (NANNARONE et al., 2015).

Embora a lidocaína IV não tenha sido extensivamente testada na espécie em questão, o seu uso como alternativa aos anti-inflamatórios durante a abordagem inicial promoveu bem-estar suficiente para que o animal passasse a ingerir água e alimento no primeiro dia, havendo ressurgimento dos movimentos ruminais. Vale ressaltar que a terapia com opioides foi desconsiderada como alternativa, devido à possibilidade de causar hipomotilidade gastrointestinal com agravamento da atonia ruminal (HOLZER, 2010).

Diante do exposto, depreende-se que a suspensão do uso de AINE e a IC de lidocaína possivelmente foram os fatores contribuintes para que o ovino voltasse a apresentar movimentação ruminal. $\mathrm{Na}$ espécie equina, a lidocaína tem sido reportada como analgésico, antihiperalgésico e promotor de melhora da função gastrintestinal pós-cirúrgica, além de propriedades anti-inflamatórias associadas à melhora da reabilitação (GOZALO-MARCILLA et al., 2014).

A normalização das variáveis cardiorrespiratórias associada ao interesse imediato por alimento, somente após a infusão da lidocaína, permite inferir que o anestésico local ofertou analgesia e contribuiu para a evolução positiva do quadro clínico. Tal achado encontra suporte na literatura, visto que, embora o uso de agentes anestésicos locais pela via parenteral em ruminantes ainda seja pouco reportado, a lidocaína em uso isolado ou associado a outros fármacos, em outras espécies, demonstrou resultados satisfatórios no controle da dor, reportando-se estabilidade simpática após uso IV (BAKAN et al. 2015; DE OLIVEIRA et al., 2010; DOHERTY et al. 2007, LAURENT, 2008).

Embora o mecanismo de ação por esta via não seja completamente elucidado, sabe-se, historicamente, que produz analgesia para dores agudas, crônicas, neuropáticas e inflamatórias em pacientes humanos (GLAZER \& PORTENOY, 1991; SOUZA \& KRAYCHETE, 2014). A administração intravenosa da lidocaína no período perioperatório sucede alívio da dor pós-cirúrgica e redução no consumo de opiodes (VENTHAM et al. 2015). No intra-operatório, o uso intravenoso guarda evidências na redução do consumo de anestésicos inalatórios e opioides na espécie canina (ORTEGA \& CRUZ, 2011; MATSUBARA et al., 2009), na espécie equina (NANNARONE et al., 2015) e em bezerros (ARAUJO et al., 2014), bem como em caprinos anestesiados com isofluorano, quando associada à cetamina (DOHERTY et al., 2007) e em ovelhas, quando associada à morfina e à cetamina (BEIER et al. 2014).

A exemplo de outros estudos, na situação clínica em pauta, o uso da lidocaína como alternativa analgésica mostrou-se igualmente promissor. Vale ressaltar que, conforme já esperado, não houve evolução suficiente do quadro renal, uma vez que a persistência da obstrução impediria resultados mais satisfatórios. Em face ao exposto, com a melhora do estado geral, o animal foi sequencialmente encaminhado à uretrostomia. 


\section{CONCLUSÕES}

Conclui-se que infusão de lidocaína na dose proposta contribuiu para o alívio da dor, retorno da atividade ruminal e melhora do estado geral do animal sendo, portanto, recomendada como recurso analgésico alternativo em ovinos com insuficiência renal e dor de intensidade moderada refratária ao uso de AINE.

\section{REFERÊNCIAS}

ARAUJO, M. A.; DIAS, B. P.; BOVINO, F.; DESCHK, M.; ABIMUSSI, C. J. X; OLIVA, V. N. L.; RODRIGUES, C. A.; SANTOS, P. S. P. Cardiovascular effects of a continuous rate infusion of lidocaine in calves anesthetized with xylazine, midazolam, ketamine and isoflurane. Veterinary anaesthesia and analgesia, v. 41, n. 2, p. 145152, 2014.

ARAUJO, M. C.; SINNOTT, C. J.; STRICHARTZ, G. R. Multiple phases of relief from experimental mechanical allodynia by systemic lidocaine: responses to early and late infusions. Pain, v. 103, n. 1, p. 21-29, 2003.

BAKAN, M.; UMUTOGLU, T.; TPOUZ, U.; UYSAL, H.; BAYRAM, M.; KADIOGLU, H.; SALIHOGLU, Z. Opioid-free total intravenous anesthesia with propofol, dexmedetomidine and lidocaine infusions for laparoscopic cholecystectomy: a prospective, randomized, double-blinded study. Revista Brasileira de Anestesiologia v. 65, n. 3, p. 191-199, 2015.

BEIER, S. L.; ROSA, A. C.; OLESCOVICZ ,N.; MATTOSO, C. R. S.; DALLABRIDA, A. L.; MORAES, A. N. Effects of morphine-lidocaine-ketamine combination on cardiopulmonary function and isoflurane sparing in sheep. Semina: Ciências Agrárias, v. 35, n. 5, p. 2527-2538, 2014.

DE OLIVEIRA, C. M. B.; ISSY, A. M.; SAKATA, R. K. Lidocaína por via venosa intraoperatória. Revista Brasileira de Anestesiologia, v. 60, n. 3, p. 183-187, 2010.

DOHERTY, T.; REDUA M. A.; QUEIROZ-CASTRO, P., EGGER, C.; COCKER S. K.; ROHRBACH, B. W. Effect of intravenous lidocaine and ketamine on the MAC of isoflurane in goats. Veterinary Anaesthesia and Analgesia, v. 34, n. 2, p. 125-131, 2007.

DUKE, T. Partial intravenous anesthesia in cats and dogs. The Canadian Veterinary Journal, v. 54, n. 3, p. 276, 2013.

FITZPATRICK, J.; SCOTT, M.; NOLAN, A. Assessment of pain and welfare in sheep. Small Ruminant Research, v. 62, n. 1, p. 55-61, 2006.

GALATOS, A. D. Anesthesia and analgesia in sheep and goats. Veterinary Clinics of North America: Food Animal Practice, v. 27, n. 1, p. 47-59, 2011.

GLAZER, A. B.; PORTENOY, M. K. Systemic Local Anesthetics in Pain Control. Journal of Pain and Symptom Management, v. 6, n. 1, p. 30-39, 1991. 
GOLZARI, S. E.; SOLEIMANPOUR, H.; MAHMOODPOOR, A.; SAFARI, S.; ALA, A. Lidocaine and Pain Management in the Emergency Department: A Review Article. Anesthesiology and pain medicine, v. 4, n. 1, 2014.

GOZALO-MARCILLA, M.; GASTHUYS, F.; SCHAUVLIEGE, S. Partial intravenous anaesthesia in the horse: a review of intravenous agents used to supplement equine inhalation anaesthesia. Part 1: lidocaine and ketamine. Veterinary anaesthesia and analgesia, v. 41, n. 4, p. 335-345, 2014.

HOLZER, P. Opioid antagonists for prevention and treatment of opioid-induced gastrointestinal effects. Current Opinion in Anesthesiology, v. 23, n. 5, p. 616-622, 2010.

LAURETTI, G. R. Mecanismos Envolvidos na Analgesia da Lidocaína por Via Venosa. Revista Brasileira de Anestesiologia, v. 58, p. 280-286, 2008.

LIZARRAGA, I.; CHAMBERS, J. P. Use of analgesic drugs for pain management in sheep. New Zealand veterinary journal, v. 60, n. 2, p. 87-94, 2012.

MATSUBARA, L. M.; OLIVA, V. N. L; GABAS, D. T.; OLIVEIRA, G. C. V.; CASSETARI, M. L. Effect of lidocaine on the minimum alveolar concentration of sevoflurane in dogs. Veterinary anaesthesia and analgesia, v. 36, n. 5, p. 407-413, 2009.

MCCLEANE, G. Intravenous lidocaine: an outdated or underutilized treatment for pain? Journal of palliative medicine, v. 10, p. 798-805, 2007.

NANNARONE, S.; CENANI, A.; GIALLETTI, R.; PEPE, M. Clinical comparison of two regimens of lidocaine infusion in horses undergoing laparotomy for colic. Veterinary anaesthesia and analgesia, v. 42, n. 2, p. 150-156, 2015.

ORTEGA, M.; CRUZ, I. Evaluation of a constant rate infusion of lidocaine for balanced anesthesia in dogs undergoing surgery. The Canadian Veterinary Journal, v. 52, n. 8, p. 856, 2011.

PLUMMER, P. J.; SCHLEINING, J. A. Assessment and management of pain in small ruminants and camelids. Veterinary Clinics of North America: Food Animal Practice, v. 29, n. 1, p. 185-208, 2013.

SMITH, G. W.; DAVIS, J. L.; TELL, L. A.; WEBB, A. I.; RIVIERE, J. E. Extralabel use of nonsteroidal anti-inflammatory drugs in cattle. Journal of the American Veterinary Medical Association, v. 232, n. 5, p. 697-701, 2008.

SOUZA, M. F.; KRAYCHETE, D. C. A ação analgésica da lidocaína intravenosa no tratamento da dor crônica: uma revisão de literatura. Revista Brasileira de Reumatologia, v. 54, n. 5, p. 386-392, 2014. 
VALVERDE, A.; GUNKEL, C. I. Pain management in horses and farm animals. Journal of Veterinary Emergency and Critical Care, v. 15, n. 4, p. 295307, 2005.

VENTHAM, N. T.; KENNEDY, E. W.; BRADY, R.R.; PATERSON, H.M.; SPEAKE, D.; FOO, I.; FEARON, K. C. H. Efficacy of intravenous lidocaine for postoperative analgesia following laparoscopic surgery: a meta-analysis. World Journal of Surgery, v. 39, n. 9, p. 2220-2234, 2015.

VESAL, N.; SPADAVECCHIA, C.; STEINER, A.; KIRSCHER, F.; LEVIONNOIS, O. L. Evaluation of the isoflurane-sparing effects of lidocaine infusion during umbilical surgery in calves. Veterinary anaesthesia and analgesia, v. 38, n. 5, p. 451-460, 2011.

WINTER, A. C. Lameness in sheep. Small Ruminant Research, v. 76, n. 1, p. 149153, 2008. 